\title{
Modelling and assessment of ROV capacity within an autonomous offshore intervention system
}

\author{
Chenyu Zhao, PR Thies, L Johanning \\ University of Exeter, $U K$ \\ S Tobin, \\ L3Harris Technologies, UK
}

\begin{abstract}
The Autonomous Surface Vehicles/ Remotely Operated Vehicles (ASV/ROV) system has received significant industrial and academic attention for offshore applications because of its low-risk operations in harsh and potentially dangerous conditions. In the ASV/ROV system, an ASV is generally regarded as the mother ship, launching and recovering the ROV via the tether management system (TMS). This study discusses the hydrodynamic and mission performance of three potential ROVs in an ASV/ROV system to support the decision making for ROV selection. The considered ROVs have different physical properties, dive characteristics and payload capabilities. This paper describes and applies a coupled hydrodynamic model to assess the capability of the different types of ROV in an automated system. For the ROVs, a nonlinear thrust control strategy is employed to reach the target The ROV capability is tested against different current velocities and water depths. The results show that the ROV with the smallest normalized thrust, i.e. max vertical thrust/the total payload, requires the lowest umbilical payout rate to reach the target. The results also show that the tidal current capacity of the ROV, i.e. the current the ROV is able to overcome, depends on the target depth and the normalized thrust. The tidal current capacity increases with a smaller target water depth and a larger normalized thrust. The methods to determine capacity envelopes for ROVs in automated systems will be useful for practitioners and researcher working on autonomous offshore systems.
\end{abstract}




\section{INTRODUCTION}

Ocean exploration has made significant progress in recent years, but it is still facing numerous challenges such as high risk and cost (Raineault and Flanders, 2019; Verfuss et al., 2019; Wright, 2020). The ASV/ROV (Autonomous Surface Vehicles/ Remotely Operated Vehicles) system, without direct physical human involvement, can address these issues during, especially in harsh and potentially dangerous environments, which require inspections or interventions of assets. The history of the ASV/ROV system dates back to World War II, with first military applications(Kumar and Kurmi, 2018). Since then its application has been extended into civilian areas such as scientific research, environmental missions and ocean resource exploration(Conte et al., 2017; Kumar and Kurmi, 2018; Sivčev et al., 2018; Trslic et al., 2020; Verfuss et al., 2019). As a crucial part of the ASV/ROV system, the ROV is always connected with ASV by an umbilical which supplies the power and data signal connectivity(Sarda and Dhanak, 2016). Based on its purpose, the ROV is generally classified into the inspection-class and interventionclass devices. For deep-water missions (usually from $200 \mathrm{~m}$ to $6000 \mathrm{~m}$ ), the intervention-class ROV is a common choice because of its relatively larger mass and more robust structure (Capocci et al., 2017). For most other and shallow water tasks, the inspectionclass ROVs can meet the requirement with lower cost and smaller dimensions (see Table 1).

Table 1 Cases applications for inspection-class ROVs (Capocci et al., 2017)

\begin{tabular}{ll}
\hline Applications & Cases \\
\hline Environmental study & Costal monitoring, Hab- \\
& itat monitoring, Pollu- \\
& tion assessments, Hull \\
& inspections, unexploded \\
& (UXO) ordnance sur- \\
& veys, Contraband detec- \\
& tion
\end{tabular}

Sciences

Seabed investigation, Marine life studies, Water and sediment sampling

Offshore oil and gas Pipe and structure inspection, visual leak detection, Diver buddy operations

Marine renewable en- Structure inspection ergy

Generally, the umbilical between the ASV and ROV is controlled by the launch and recovery system
(LARS). For example, a system without dedicated docking station is equipped with a video ray microROV to launch and recover the ROV directly (Capocci et al., 2017). As a result, the LARS could be subject to strong coupled effects between the ASV and the ROV, which are usually not considered in most studies (Trslic et al., 2020). In a recent study (Zhu et al., 2008), the coupled effects between the umbilical and ROV are discussed while the ASV's effects are ignored. In their study, the ROV is regarded as a 6-DOF lumped buoy and umbilical connected with the ROV has a constant length $(300 \mathrm{~m})$ without any pay-out/in speed. Both physical and numerical results showed that the tidal current could significantly affect the umbilical tension.

This present study applies a fully nonlinear numerical model to explore the capacity of the ROV in a coupled ROV/ASV system. This model is based on the potential flow theory and includes both a frequency-model model and a time-domain model. The external forces are modelled through Python codes to limit the motion amplitude of the ROV during the launch and recovery stages. The paper is organised as follows: Section 2 lays out the fully coupled model, including its governing equation and configurations; Section 3 introduces the tidal current capacity results of three different ROVs. Section 4 discusses the main findings. Section 5 concludes with the primary outcomes and further work.

\section{NUMERICAL MODELLING}

\subsection{Model illustration}

An overview of the modelling scope is provided in Figure 1. The numerical simulation includes a frequency- and a time-domain model. The hydrodynamic forces of the ASV, are calculated as the sum of diffraction (including exciting and Froude-Krylov forces) and radiation forces, obtained by the frequency-domain boundary element method solver, namely AQWA (ANSYS, 2010). The simplified CAD drawing used as an input for AQWA to calculate the Response Amplitude Operators (RAOs) is also shown in Figure 1. The physical properties of three ROVs and umbilical configurations are implemented in a fully coupled nonlinear hydrodynamic time-domain [Orcaflex (Manual, 2012)] to estimate the response, forces and loads experienced by the vessel, ROV and the umbilical cable, shown in Figure 2. The Python code provides the external force to control the ROV motion to complete the launch and recovery missions. 


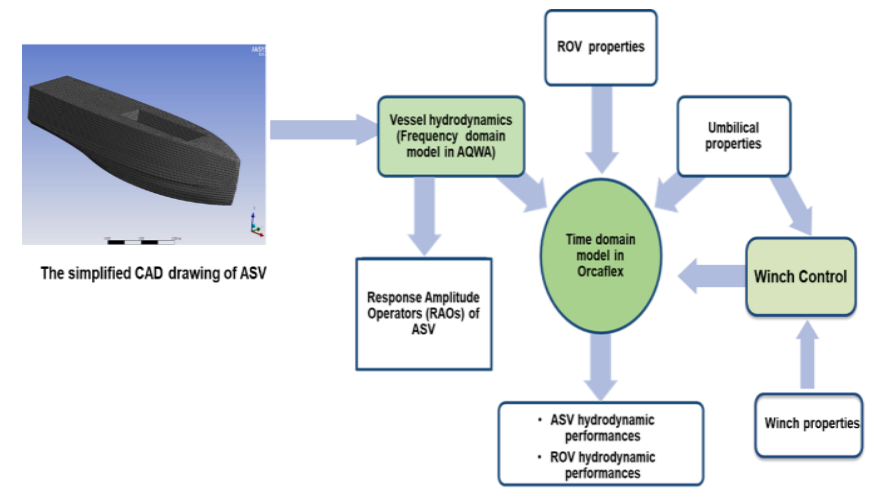

Figure 1: The overview of the numerical model, including a frequency- and a time-domain model

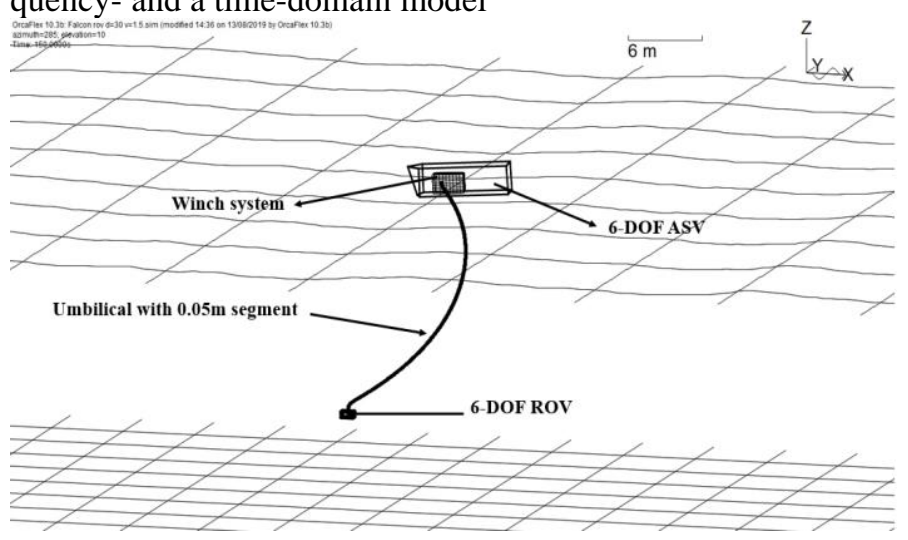

Figure 2: Wire frame model setup of the coupled time-domain model in Orcaflex

\subsection{Governing equation}

The analytical model of the ASV is illustrated in Figure 3 , and its governing equation are presented as follows:

$[M+m]\{\ddot{\xi}\}+[B]\{\dot{\xi}\}+\{[K]+[C]\}\{\xi\}+\left[F_{u}\right]=\left[F_{e}\right]$

(1)

where the $[M+m]$ is the mass (including the added mass $m$ ) matrix, $[B]$ is the radiation damping matrix, $[K]$ is the hydrostatic stiff matrix, $[C]$ is the control force matrix, $\xi$ is the ASV's motion equation, $\left[F_{u}\right]$ is the umbilical's force matrix and $F_{e}$ is the wave exciting force matrix.

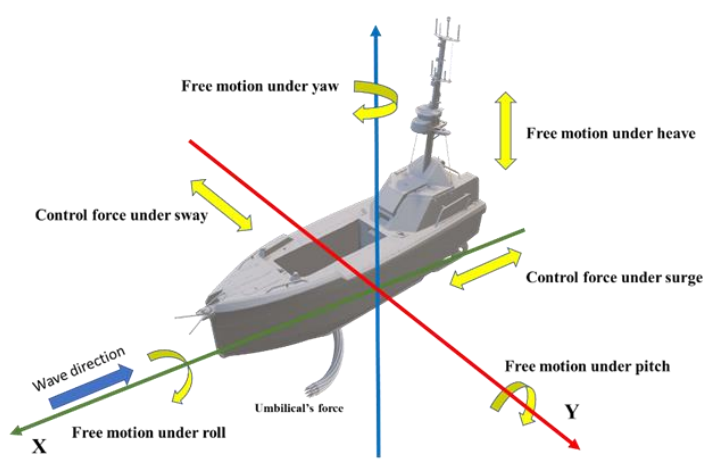

Figure 3: Analytical ASV model, control forces are applied in sway and surge modes.
$F_{u}$ includes the umbilical's drag force and tensions. The standard Morrison equations calculate the drag forces. The fluid velocity relative to the line is split into its components $v_{n}$ and $v_{z}$ normal and parallel to the line axis. The drag force normal to the line axis is then determined by $v_{n}$ and its $x$ and $y$ components $v_{x} v_{x}$. the drag force parallel to the line axis is determined by $v_{z}$

$$
\begin{aligned}
& f_{D x}=\frac{1}{2} p \rho d_{n} l C_{D x} v_{x}\left|v_{n}\right| \\
& f_{D y}=\frac{1}{2} p \rho d_{n} l C_{D y} v_{y}\left|v_{n}\right| \\
& f_{D z}=\frac{1}{2} p \rho d_{n} l C_{D z} v_{z}\left|v_{n}\right|
\end{aligned}
$$

The tension is presented as a spring:

$$
F_{\text {tension }}=K_{u} \Delta l
$$

where $K_{u}$ is the spring coefficient, $\Delta l$ is the deformation of the umbilical.

During the launch phase, the ROV has a relatively small displacements and always operates in areas far from the water surface, the wave forces will be ignored during the calculation. The total forces on the ROV can be divided into control forces (moments), current forces, umbilical's forces and the hydrostatic forces.

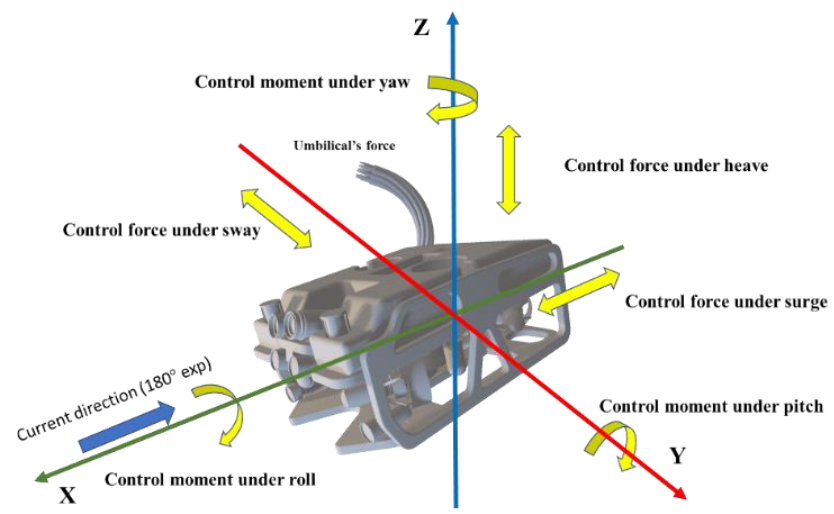

Figure 4: The analytical model of ROV: The control forces and moments are applied under all 6 DOFs.

The ROV is initially driven with full thrusters so that it can quickly reach a depth to prevent it from hitting the ASV. Then, the control method on the $\mathrm{x}$, y direction offers a soft way for the ROV's propeller force to increase in function of the depth of ASV:

$$
\left.\begin{array}{l}
F_{l x}=\left(x_{\text {target }}-y_{R O V}\right) * k_{l x} * \log _{2}\left(z_{A S V}-z_{R O V}\right) \\
F_{l y}=\left(y_{\text {target }}-y_{R O V}\right) * k_{l y} * \log _{2}\left(z_{A S V}-z_{R O V}\right.
\end{array}\right)
$$

When $F_{l x}$ and $F_{l y}$ are larger than the maximum thrust of ROV, the maximum thrusts will replace them. 
The z-direction force $F_{l z}$ includes a constant component $C_{z}$ that permits to approach the target plus a Gaussian function around the target allowing the force to increase a lot when approaching the target in order to maintain the ROV's depth. Similar to the $F_{l x}$ and $F_{l y}, F_{l z}$ will still be limited by the maximum ROV thrust.

$F_{l z}=C_{z}+k_{l z}\left(\frac{1}{5} e^{-\left(z_{A S V}-z_{R O V}\right)^{2}}\right)$

where $x_{\text {target }}, y_{\text {target }}, z_{\text {target }}$ are the target in each direction; $x_{A S V}, y_{A S V}, z_{A S V}$ are the displacement of ASV in each direction; $x_{R O V}, y_{R O V}, z_{R O V}$ are the displacement of ROV in each direction; $k_{l x}, k_{l y}, k_{l z}$ are the launch control coefficients in each direction.

\section{RESULTS}

The wave condition is defined as a JONSWAP spectrum with the significant wave height $\mathrm{Hs}=1 \mathrm{~m}$ and peak wave period $\mathrm{Tp}=5 \mathrm{~s}$. The tidal current is defined by the power-law method, and its direction is fixed and does not vary with depth. The current speed $S_{c}$ varies with the water depth Figure 5. The direction of the tidal current is heading to the ROV.

$S_{c}=S_{b}+\left[\left(S_{f}-S_{b}\right) \frac{z-z_{b}}{z_{f}-z_{b}}\right]^{1 / p}$

where $S_{f}$ and $S_{b}$ are the current speeds at the surface and the seabed, respectively; $p$ is the powerlaw exponent; $z_{f}$ is the $\mathrm{z}$ - coordinate of the still water level; $z_{b}$ is the $\mathrm{z}$ - coordinate of the seabed.

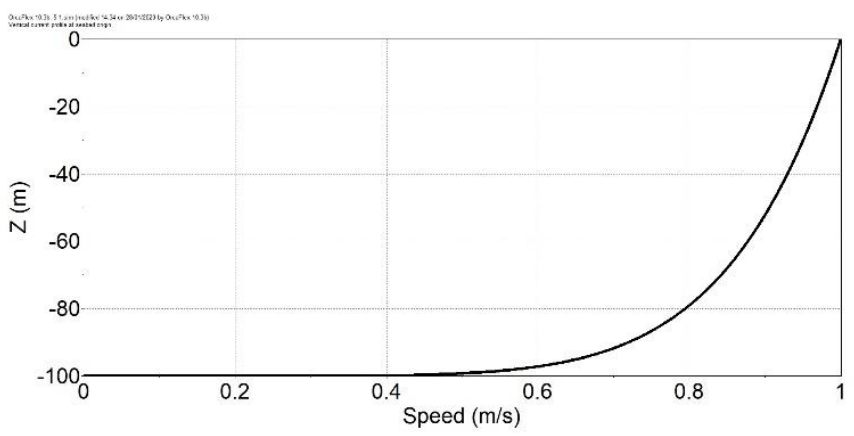

Figure 5: The speed of the tidal current with $100 \mathrm{~m}$ water depth, the speed on the water surface is $1 \mathrm{~m} / \mathrm{s}$

In the ROV/ASV system, three ROVs and one ASV (Table 2) are used. A recent review has identified potentially suitable ROVs for the considered ASV platform (Sivčev et al., 2018). This study chose three ROVs, based on commercial considerations. These three ROVs have different dimensions and capabilities, retrieved from their publicl available specification sheets (shown in Table 3). The $\mathrm{x}$ - and $\mathrm{y}$ - coordinates of target positions are identical $(10 \mathrm{~m}, 0 \mathrm{~m})$ while the z-coordinates are $20 \mathrm{~m}, 40 \mathrm{~m}$, and $100 \mathrm{~m}$ for each water depth. The pay-out rate of the umbilical for ROV A, ROV B and ROV C are configured to be $0.2 \mathrm{~m} / \mathrm{s}, 0.4 \mathrm{~m} / \mathrm{s}$ and $0.4 \mathrm{~m} / \mathrm{s}$ to ensure the ROV can reach the target position timely and the curvature of the umbilical does not exceed the limitations.

Table 2 the properties of the CW7

\begin{tabular}{lc}
\hline Property & Value (unit) \\
\hline Length & $7.2 \mathrm{~m}$ \\
Beam & $2.3 \mathrm{~m}$ \\
Draft & $0.9 \mathrm{~m}$ \\
Weight (without pay- & $4280 \mathrm{~kg}$ \\
load) &
\end{tabular}

Table 3 the properties of three ROVs

\begin{tabular}{llll}
\hline Property & A & B & C \\
\hline $\begin{array}{l}\text { Dimen- } \\
\text { sion(L*W }\end{array}$ & $1000 * 600 *$ & $800 * 600 *$ & $1000 * 600 *$ \\
$* \mathrm{H}) \mathrm{mm}$ & & 485 & 500 \\
$\begin{array}{l}\text { Total pay- } \\
\text { load }\end{array}$ & $74 \mathrm{~kg}$ & $100 \mathrm{~kg}$ & $97 \mathrm{~kg}$ \\
$\begin{array}{l}\text { Thrust } \\
\text { Lateral }\end{array}$ & $0.49 \mathrm{kN}$ & $0.715 \mathrm{kN}$ & $0.52 \mathrm{kN}$ \\
$\begin{array}{l}\text { Thrust } \\
\text { Vertical }\end{array}$ & $0.1274 \mathrm{kN}$ & $0.441 \mathrm{kN}$ & $0.255 \mathrm{kN}$ \\
$\begin{array}{l}\text { Max lat- } \\
\text { eral } 6.62 \mathrm{~N} / \mathrm{kg}\end{array}$ & $7.15 \mathrm{~N} / \mathrm{kg}$ & $5.36 \mathrm{~N} / \mathrm{kg}$ \\
thrust/to- \\
$\begin{array}{l}\text { tal pay- } \\
\left.\text { load ( } R_{1}\right)\end{array}$
\end{tabular}

Four tidal currents (surface velocity: $1.25 \mathrm{~m} / \mathrm{s}, 1.5 \mathrm{~m} / \mathrm{s}$, $1.75 \mathrm{~m} / \mathrm{s}, 2 \mathrm{~m} / \mathrm{s}$ ) are used to discuss the capacity for each ROV. Table 4 shows the maximum current speed that allows ROV to reach the target position. For all three ROVs, the tidal current capacity decreases when they need to reach a deeper position. ROV A and B have identical current capacity during all cases. The current capacity of the ROV $\mathrm{C}$ is the weakest and seems to be the most sensitive to the target depth. 
Table 4 the tidal current capacity of three ROVs

\begin{tabular}{llll}
\hline Target depth & A & B & C \\
\hline $20 \mathrm{~m}$ & $2 \mathrm{~m} / \mathrm{s}$ & $2 \mathrm{~m} / \mathrm{s}$ & $1.75 \mathrm{~m} / \mathrm{s}$ \\
$40 \mathrm{~m}$ & $1.75 \mathrm{~m} / \mathrm{s}$ & $\begin{array}{l}1.75 \\
\mathrm{~m} / \mathrm{s}\end{array}$ & $1.5 \mathrm{~m} / \mathrm{s}$ \\
& & $\begin{array}{l}1.75 \\
\mathrm{~m} / \mathrm{s}\end{array}$ & $1.25 \mathrm{~m} / \mathrm{s}$ \\
\hline
\end{tabular}

Figure 6 presents the tension on the umbilical during the $100 \mathrm{~m}$ case. The results determined the positive correlation between the maximum vertical thrust/total payload $R_{2}$ and the umbilical tension (for both mean and maximum value). The differences in the STD tension between these three ROV are small; the amplitude of ROV C is just slightly larger than the other two ROVs. It should be noticed that the maximum tension appears at the connection point between ASV and umbilical during the first 20 seconds of the launch during all cases.

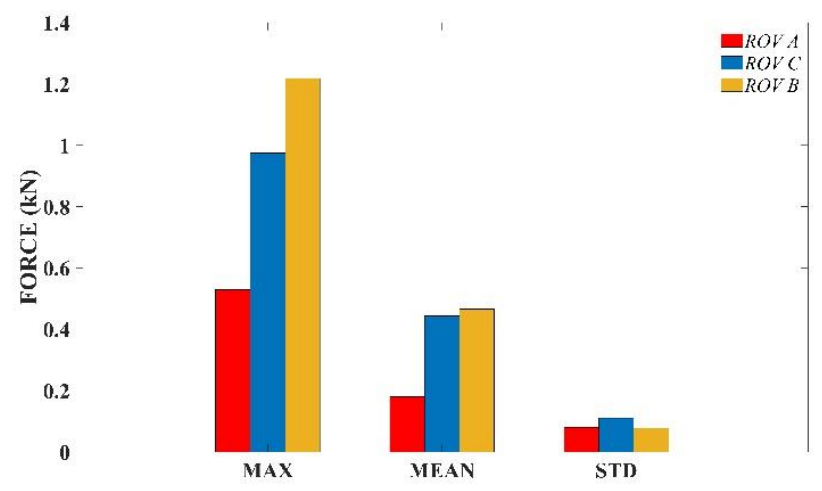

Figure 6: The umbilical tension of each ROV during $140 \mathrm{~m}$ cases, the maximum tension appears time, ROV A 12s, ROV B $10 \mathrm{~s}, \mathrm{ROV} \mathrm{C} 11 \mathrm{~s}$.

\section{DISCUSSIONS}

\subsection{Tidal current capacity}

According to Table 3, the tidal current capacity of ROV is mainly dependent on the maximum lateral thrust/total payload value, $R_{1}$ and the Target depth of the ROV. The larger $R_{1}$ means the lateral thrust per unit mass is more significant. Therefore, the ROV can overcome a stronger tidal current. When the target depth of the ROV is larger, the ROV needs a longer umbilical. The drag force caused by the tidal current will be more significant, resulting in a decrease of ROV's current capacity. If the tidal current capacity of the ROV is considered without taking the umbilical drag into consideration, it would be constant. For example, when the target depth of ROV C is less than $70 \mathrm{~m}$, its current capacity is at least 1.5 $\mathrm{m} / \mathrm{s}$. While the capacity reduces to be $1.25 \mathrm{~m} / \mathrm{s}$ if the target depth is $100 \mathrm{~m}$ (See Figure 7). Additionally, the higher current tidal current could decrease the velocity of ROV to get the target.

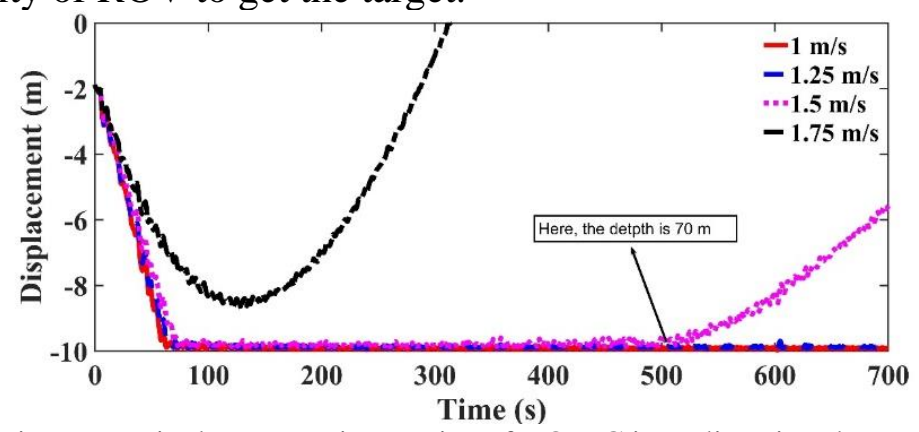

Figure 7: Displacement time series of ROV C in X-direction during the $100 \mathrm{~m}$ case.

\subsection{Umbilical tension}

Considering the net mass (mass in the water) of the umbilical, the maximum tension appears at the connection point on the ASV rather than the point on the ROV. The time of appearance of the maximum tension warrants some consideration. To explore the occurrence, the ROV B is placed into various water depths as its initial conditions. The length of the umbilical is only based on this initial position of ROV without any pay-out/in rate. The results show that the maximum tension maintains a high level (larger than $1 \mathrm{kN}$ ) when the length of umbilical is less than $20 \mathrm{~m}$ (see Figure 8). When the umbilical length exceeds $30 \mathrm{~m}$, the maximum tension is almost independent from the length. It is believed that the maximum tension of the umbilical is mainly caused by the sudden relative motion between ASV and ROV rather than the umbilical mass. This relative motion could be buffered when the umbilical length is long enough (here $30 \mathrm{~m}$ ). These results demonstrate that the coupled effects between ASV and ROV could significantly influence the umbilical tension.

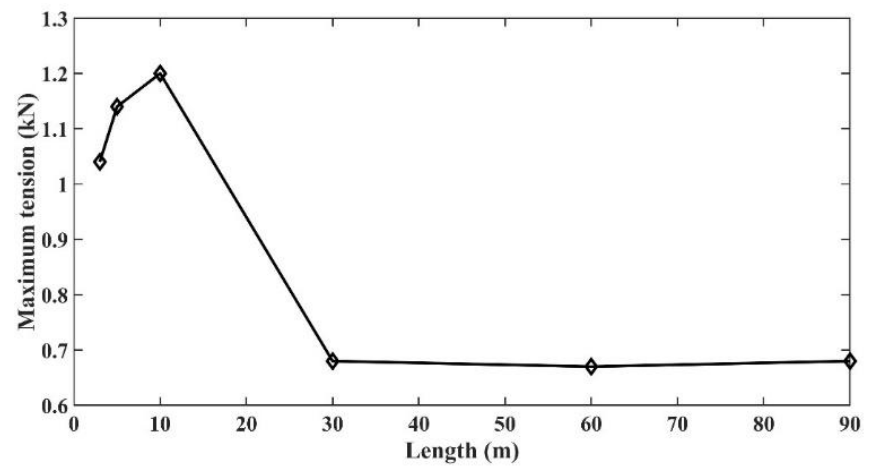

Figure 8: Maximum tension under different umbilical length

\section{CONCLUSION}

This study explored the capacity of ROV in an autonomous offshore intervention system based on a fully coupled numerical model. The results indicate that the coupled effects caused by the ASV, the ROV, and the umbilical significantly influence the capacity of 
the ROV. Comparing three different ROVs, it is determined that the ROV with a larger maximum lateral thrust/total payload, $R_{1}$, has a higher tidal current capacity. Because of the drag force on the umbilical, the tidal current capacity of the ROV decreases with a deeper target.

Additionally, the results of umbilical tension found that the larger max vertical thrust/total payload value, $R_{2}$, increase the maximum and mean tension. Comparing umbilical tension with a range of lengths suggests that the sudden relative motion between the ASV and $\mathrm{ROV}$ is the main reason for the maximum tension events modelled. In future research, more control methods of umbilical pay-out/in will be explores to reduce the tension on the umbilical and to enhance the stabilisation of the ROV motion.

\section{ACKNOWLEDGEMENT}

This has received funding through the project " $\mathrm{Au}$ tonomous Robotic Intervention System For Extreme Maritime Environments (ARISE) Stage 2", as part of the Industry Strategy Challenge Fund (ISFC) funded by Innovate UK (UKRI), Project Reference: 104831

\section{REFERENCES}

ANSYS, A., 2010. Uer's Manual. Ansys Inc.

Capocci, R., Dooly, G., Omerdić, E., Coleman, J., Newe, T., Toal, D., 2017. Inspection-class remotely operated vehicles-A review. Journal of Marine Science and Engineering 5 (1), 13.

Conte, G., Scaradozzi, D., Mannocchi, D., Ciuccoli, N., 2017. Field Test of an Integrated ASV/ROV Platform, The 27th International Ocean and Polar Engineering Conference. International Society of Offshore and Polar Engineers.

Kumar, A., Kurmi, J., 2018. A REVIEW ON UNMANNED WATER SURFACE VEHICLE. International Journal of Advanced Research in Computer Science 9 (Special Issue 2), 95. Manual, O., 2012. Online at http://www. orcina. com/SoftwareProducts/OrcaFlex/Documentation. OrcaFlex. pdf.

Raineault, N.A., Flanders, J., 2019. New frontiers in ocean exploration: The E/V Nautilus, NOAA Ship Okeanos Explorer, and R/V Falkor 2018 field season.

Sarda, E.I., Dhanak, M.R., 2016. A USV-Based automated launch and recovery system for AUVs. IEEE journal of oceanic engineering 42 (1), 37-55.

Sivčev, S., Coleman, J., Omerdić, E., Dooly, G., Toal, D., 2018. Underwater manipulators: A review. Ocean Engineering 163, 431-450.

Trslic, P., Rossi, M., Robinson, L., O’Donnel, C.W., Weir, A., Coleman, J., Riordan, J., Omerdic, E., Dooly, G., Toal, D., 2020. Vision based autonomous docking for work class ROVs. Ocean Engineering 196, 106840.

Verfuss, U.K., Aniceto, A.S., Harris, D.V., Gillespie, D., Fielding, S., Jiménez, G., Johnston, P., Sinclair, R.R., Sivertsen, A., Solb $\varnothing$, S.A., 2019. A review of unmanned vehicles for the detection and monitoring of marine fauna. Marine pollution bulletin 140, 17-29.
Wright, R.G., 2020. Unmanned and Autonomous Ships: An Overview of MASS. Routledge.

Zhu, K.-q., Zhu, H.-y., Zhang, Y.-s., Jie, G., MIAO, G.-p., 2008. A multi-body space-coupled motion simulation for a deep-sea tethered remotely operated vehicle. Journal of Hydrodynamics, Ser. B 20 (2), 210-215. 